\title{
Modeling the Influence of the Penetration Channel's Shape on Plasma Parameters When Handling Highly Concentrated Energy Sources
}

\author{
Dmitriy N. Trushnikov, ${ }^{1}$ Ekaterina S. Salomatova, ${ }^{1}$ Igor I. Bezukladnikov, ${ }^{2}$ \\ Igor L. Sinani, ${ }^{1}$ and K. P. Karunakaran ${ }^{3}$ \\ ${ }^{1}$ Department of Welding, Metrology, and Materials Technology, Perm National Research Polytechnic University, \\ Komsomolskiy Prospekt, No. 29, Perm 614099, Russia \\ ${ }^{2}$ Department of Automation and Telematics, Perm National Research Polytechnic University, Komsomolskiy Prospekt, \\ No. 29, Perm 614099, Russia \\ ${ }^{3}$ Department of Mechanical Engineering, Rapid Manufacturing Laboratory, Indian Institute of Technology Bombay, \\ Powai, Mumbai 400076, India
}

Correspondence should be addressed to Dmitriy N. Trushnikov; trdimitr@yandex.ru

Received 2 July 2017; Accepted 24 August 2017; Published 12 October 2017

Academic Editor: Michael Aizenshtein

Copyright (C) 2017 Dmitriy N. Trushnikov et al. This is an open access article distributed under the Creative Commons Attribution License, which permits unrestricted use, distribution, and reproduction in any medium, provided the original work is properly cited.

\begin{abstract}
In our work to formulate a scientific justification for process control methods when processing materials using concentrated energy sources, we develop a model that can calculate plasma parameters and the magnitude of the secondary waveform of a current from a non-self-sustained discharge in plasma as a function of the geometry of the penetration channel, thermal fields, and the beam's position within the penetration channel. We present the method and a numeric implementation whose first stage involves the use of a two-dimensional model to calculate the statistical probability of the secondary electrons' passage through the penetration channel as a function of the interaction zone's depth. Then, the discovered relationship is used to numerically calculate how the secondary current changes as a distributed beam moves along a three-dimensional penetration channel. We demonstrate that during oscillating electron beam welding the waveform has the greatest magnitude during interaction with the upper areas of the penetration channel and diminishes with increasing penetration channel depth in a way that depends on the penetration channel's shape. When the surface of the penetration channel is approximated with a Gaussian function, the waveform decreases nearly exponentially.
\end{abstract}

\section{Introduction}

An important problem in the development of flawless electron beam and laser welding technologies is ensuring that high-quality welds can be consistently reproduced. Work to automate the process using secondary waveform parameters has been pursued for a long time with varied success. Certain successes have been achieved using methods involving registration of the secondary X-ray radiation emitted during welding with highly concentrated energy sources. However, these methods have limitations due to the difficulty placing additional sensors within the vacuum chamber, which creates complications when used in industrial conditions. Moreover, a number of studies indicate that electron beam welding is accompanied by high-frequency processes [1-4] that often carry the most information about the thermal characteristics of the electron beam's interaction with the metal in the penetration channel. Frequencies up to $20 \mathrm{kHz}$ are inherent to these processes, which makes it possible to study them using X-ray radiation sensors. Methods based on registration of the secondary plasma current originating above the welding zone [5] are promising. Several methods have been developed to study and control electron beam welding [15] processes. Similar works in the area of laser welding have been widely conducted recently. However, we must note that the problem of controlling penetration during electron 
beam welding using the parameters of the secondary plasma current cannot yet be considered fully solved. There are no models that describe the relationship between the parameters of the secondary waveforms in the plasma. To date, there are no universal, reliable, and sufficiently responsive process control systems for making a welded joint with electron beam welding. The accumulated knowledge is frequently tentative for now.

References [1-3] describe the results of experimental studies on generating secondary current waveforms in plasma during EBW. Several hypotheses have been put forward to describe the observed phenomena. However, the proposed theories are insufficient to fully interpret the obtained results $[4,5]$. We need a detailed model of the processes occurring in the plasma. There are well-known works that estimate plasma parameters using simplified methods [610]. The authors of these works propose one-dimensional models that can be used to estimate the concentration and energy of electrons in the plasma above the EBW zone, but they do not make it possible to calculate the parameters of the non-self-sustained discharge. References [11-13] describe a two-dimensional model of the formation of the non-selfsustaining discharge in plasma during EBW. However, for practical purposes, [14] is of interest in determining how the waveform's magnitude changes during a change in the position of the beam in the penetration channel and as a function of the channel's geometric dimensions. Direct modeling requires a three-dimensional formulation, which entails unjustifiably large computational costs. This article proposes a method and numeric implementation based on a preliminary solution to the problem in a two-dimensional axisymmetric formulation with subsequent use of the results of this solution for a full description. The results obtained from this solution for measuring secondary electric current in plasma during EBW may be extended to laser welding.

\section{Description of the Model}

At the heart of the model are transfer equations for the concentration of electrons $n_{e}$ and the average electron energy in the plasma over the EBW zone [14-17]:

$$
\begin{array}{r}
\frac{\partial\left(n_{e}\right)}{\partial t}+\nabla \cdot \vec{\Gamma}_{e}=R_{e}, \\
\frac{\partial\left(n_{\varepsilon}\right)}{\partial t}+\nabla \cdot\left[-n_{\varepsilon}\left(\mu_{\varepsilon} \vec{E}\right)-D_{\varepsilon} \nabla n_{\varepsilon}\right]+e \vec{E} \cdot \vec{\Gamma}_{e}=R_{\varepsilon},
\end{array}
$$

where $\vec{\Gamma}_{e}$ is the electron stream density, $n_{\varepsilon}$ is the volumetric energy density of the electrons, $D_{\varepsilon}, \mu_{\varepsilon}$ are the electrons' diffusion coefficient and mobility coefficient for the energy, $R_{e}$ is the intensity of the electron source, $R_{\varepsilon}$ is the electrons' energy source (describes the energy loss due to inelastic collisions), and $\vec{E}$ is the electric field vector.

To describe the mass transfer of heavy plasma particles (ions, neutral unexcited and excited atoms), we use the mass transfer equation for a multicomponent mixture [18-20]:

$$
\rho \frac{\partial\left(\omega_{k}\right)}{\partial t}+\rho(\vec{u} \cdot \nabla) \omega_{k}=\nabla \cdot \vec{j}_{k}+R_{k}
$$

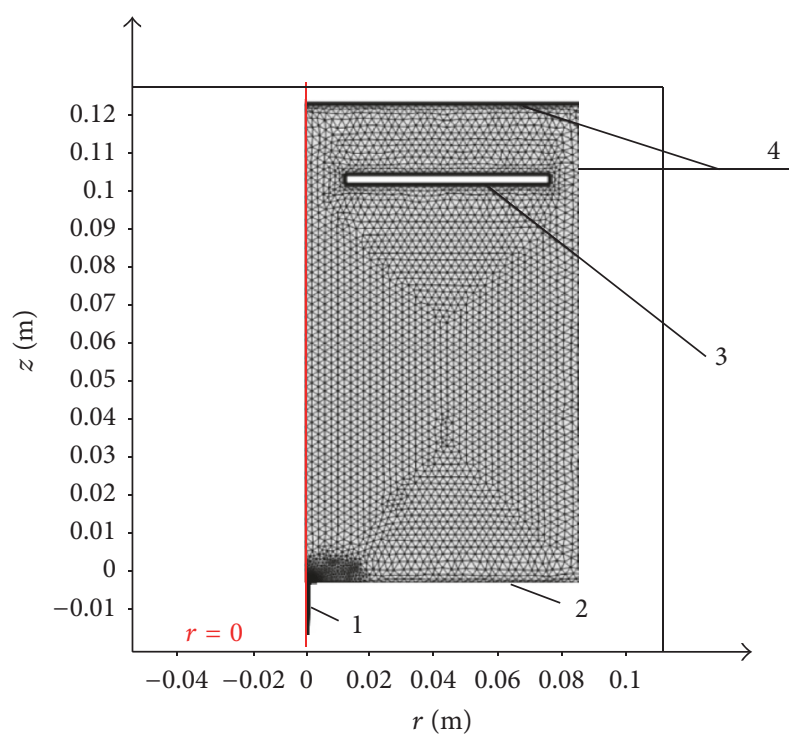

FIGURE 1: Geometry of the computational zone: 1: vapor channel; 2: surface of the sample being welded; 3: electron collector; 4: edges of the calculation zone.

where $\vec{j}_{k}$ expresses the density of the mass stream of the $k$ th component; $\omega_{k}$ is the mass concentration of the $k$ th component; $R_{k}$ is the intensity of the source of the $k$ th component; $\vec{u}$ is the average velocity vector; $\rho$ is the mixture density.

The electric field is determined from the Poisson equation

$$
\nabla^{2} U=-\frac{\rho_{0}}{\varepsilon_{0}}
$$

The plasma becomes collisionless above the penetration channel. When this happens, the diffusion coefficients in (1) go to zero and the equations degenerate into continuity equations for charge and energy.

A full system of equations and description is given in [14].

Calculations are performed in cylindrical coordinates in an axisymmetric formulation (Figure 1).

A non-self-sustaining discharge in plasma is accompanied by the emission of secondary electrons from the surface of the item (cathode). The edge conditions account for the loss of electrons on chamber walls and on the surface of the item due to random motion and thermoemissive effects [19]:

$$
-\vec{n} \cdot \vec{\Gamma}_{e}=\left(\frac{1}{2 v_{e, \mathrm{th}} n_{e}}\right)+n_{e} \mu_{e} \vec{E} \cdot \vec{n}-\vec{n} \cdot \vec{\Gamma}_{t}
$$

and for the electrons' energy flux:

$$
-\vec{n} \cdot \vec{\Gamma}_{\varepsilon}=\left(\frac{5}{6 v_{e, \mathrm{th}} n_{\varepsilon}}\right)+n_{\varepsilon} \mu_{\varepsilon} \vec{E} \cdot \vec{n},
$$

where $v_{e, \text { th }}$ is the thermal velocity of electrons, $\vec{\Gamma}_{t}=\vec{j}_{t} / e$ is the electron stream resulting from thermionic emission (only on the walls of the penetration channel), and $\vec{n}$ is the surface normal. 
TABLE 1: Plasma-chemical reactions taken into consideration.

\begin{tabular}{lcc}
\hline Reaction & Formula & Type of interaction \\
\hline 1 & $e+A \longrightarrow e+A$ & Elastic \\
2 & $e+A \longrightarrow e+A^{*}$ & Excitation \\
3 & $e+A \longrightarrow 2 e+A+$ & Ionization \\
\hline
\end{tabular}

Table 1 presents the plasma-chemical reactions taken into consideration in the calculations. As a result of the plasma's low ionization coefficient, the electrons' interactions with ions and excited atoms are ignored. The energies corresponding to each reaction are the input parameters for the model. To obtain a numeric solution, we used COMSOL 4.4 and the Plasma Module, Molecular Flow, Rarefied Flow modules. The modeling results adequately describe the patterns known from the literature and observed experimentally.

Reference [14] presents the parameters of the plasma, calculated using the presented model, not only above the welding zone but also directly in the vapor channel being formed. The mathematical model makes it possible to calculate the change in plasma parameters and the magnitude of the waveform of the secondary current in the plasma during excitation of a non-self-sustaining discharge by delivering the positive potential to the collector. Modeling the formation of this waveform is of special interest, since it is actively used when building automated control methods for EBW [21].

\section{Influence of the Penetration Channel's Shape on the Magnitude of the Secondary Emission's Waveform}

Reference [11] demonstrates that in the upper regions of the penetration channel the electric field reaches a magnitude of $10^{6} \mathrm{~V} / \mathrm{m}$. The presence of such an electric field significantly lowers the potential barrier and reduces the work to discharge electrons. In this case, the density of the emission is described by the Richardson-Schottky equation

$$
j_{t}=A T^{2} \exp \left(-e\left(\varphi-\frac{\sqrt{e E_{k}}}{k_{b} T}\right)\right),
$$

where $E_{k}$ is the intensity of the electric field.

Figure 2 presents the model's results for the electron emission's current density distributions on the walls of the penetration channel, calculated using (7) with and without the electron emission. The plots demonstrate that, despite the emission's sharp increase due to the Schottky effect in the upper regions of the penetration channel, the emission continues to tend to grow as the depth increases. This result is due to the temperature's prevailing influence on the emission. According to [21], when formulating the problem the temperature is assumed to increase in the direction of the bottom of the channel.

The magnitude of the secondary current in plasma, which is excited when a non-self-sustaining discharge is initiated, is influenced by more than just the electron emission from the walls of the penetration channel. During diffusion of slow electrons in plasma in the penetration channel, some of

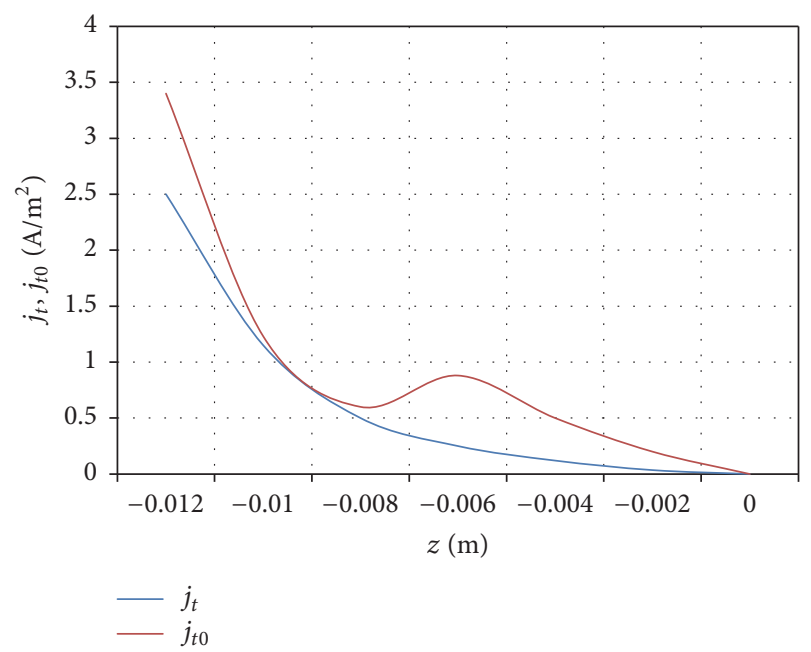

FIGURE 2: Distribution of the emission's current densities in the penetration channel during excitation of a non-self-sustaining discharge: $j_{t 0}$ is the density of the thermionic current; $j_{t}$ is the current density, accounting for the Schottky effect.

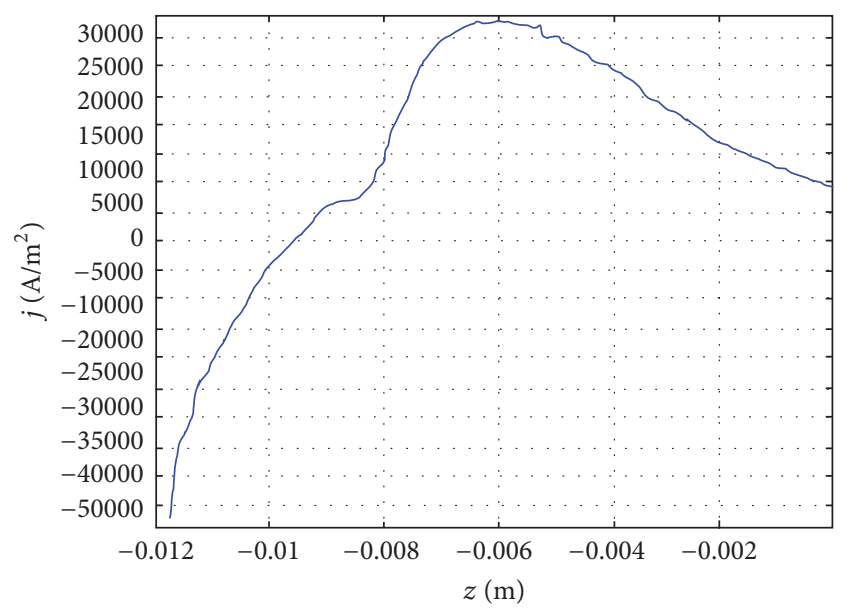

FIGURE 3: Resulting distribution of the current density on the walls of the penetration channel.

them escape into the walls of the penetration channel. Consequently, the resulting current density distribution differs from the plots in Figure 2. Figure 3 demonstrates that the influence of electron loss at the walls of the channel reduces the current density when approaching the bottom. At a depth of roughly $9 \mathrm{~mm}$, the current density changes sign for the penetration channel's specified transverse dimensions. The change in sign means that in these regions the electron stream directed at the walls begins to prevail over the stream caused by the emissive phenomenon. The fact that this effect is observed at the bottom of the channel is apparently due to the weaker electric field, which prevents its occurrence.

For practical purposes, [22] is of interest in determining how the waveform's magnitude changes during a change in the position of the beam in the penetration channel and as a function of the channel's geometric dimensions. The magnitude of the secondary current in plasma, which is 


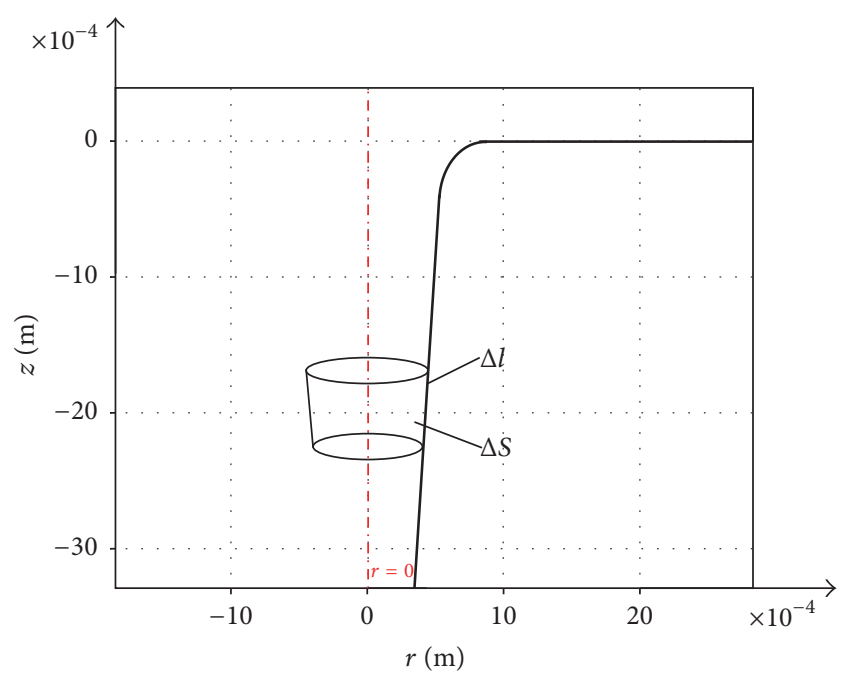

FIGURE 4: Determination of probability $p(z)$ of the secondary electrons' passage through the penetration channel.

excited when a non-self-sustaining discharge is initiated, is influenced by more than just the electron emission from the walls of the penetration channel. During diffusion of slow electrons in plasma in the penetration channel, some of them escape into the walls of the penetration channel. During oscillating EBW, the electron beam is moved along the walls of the penetration channel, causing a periodic change in the temperature field. This produces oscillations in the magnitude of the secondary current in the plasma [23].

\section{Results: Transition to a Three-Dimension Formulation}

This article proposes a numeric implementation whose first stage involves using the model to determine the statistical probability $p(z)$ of the secondary electrons' passage through the penetration channel from interaction area depth $z$, or, in other words, the ratio of the number of electrons, emitted from a certain surface element at the penetration channel's depth and reaching the collector, to the total number of electrons emitted from this element. Then, we use the discovered function $p(z)$ to numerically calculate the change in the secondary current when a distributed beam is moved about the three-dimensional penetration channel.

The value of $p(z)$ may be determined as follows. At depth $z$, a small area $\Delta S=2 \pi r \Delta l$ (Figure 4) is selected on the wall of the penetration channel. During the calculation, the response $\Delta I_{k}$ to the waveform's secondary current is increased when the emission's current density is increased in the area $\Delta S$ by $\Delta j$. The probability $p(z)$ of the secondary electrons' passage through the penetration channel from depth $z$ is determined using the following expression:

$$
p(z)=\frac{\Delta I_{k}}{\Delta j \Delta S} .
$$

Figure 5 presents the results of the calculation of the relationship between $\Delta I_{k}$ and the depth $z$ of element $\Delta S$.

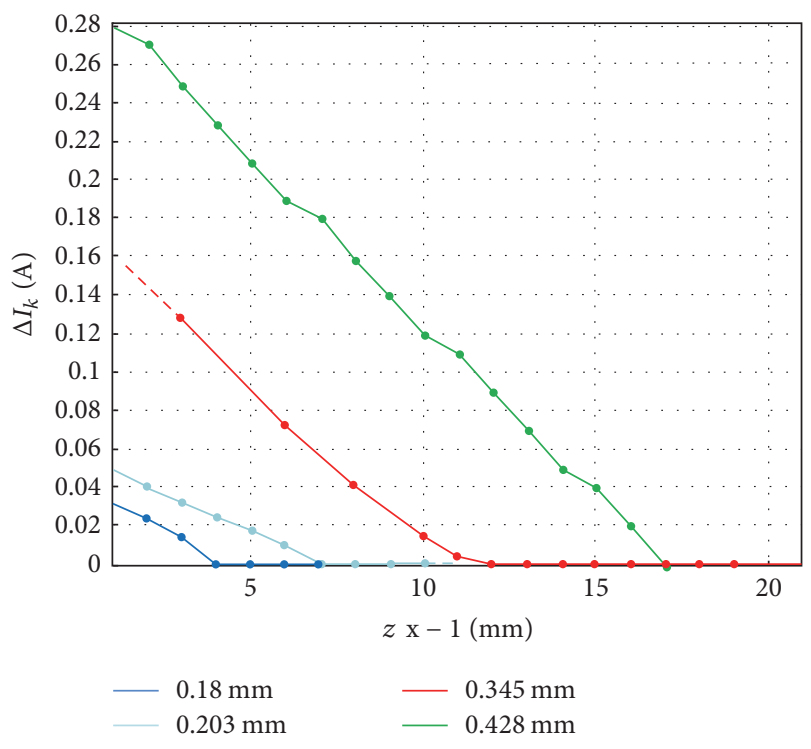

FIGURE 5: The secondary current waveform's response $\Delta I_{k}$ to changes in the emission in element $\Delta S$ as a function of depth $z$ for four penetration channels with varying transverse dimensions.

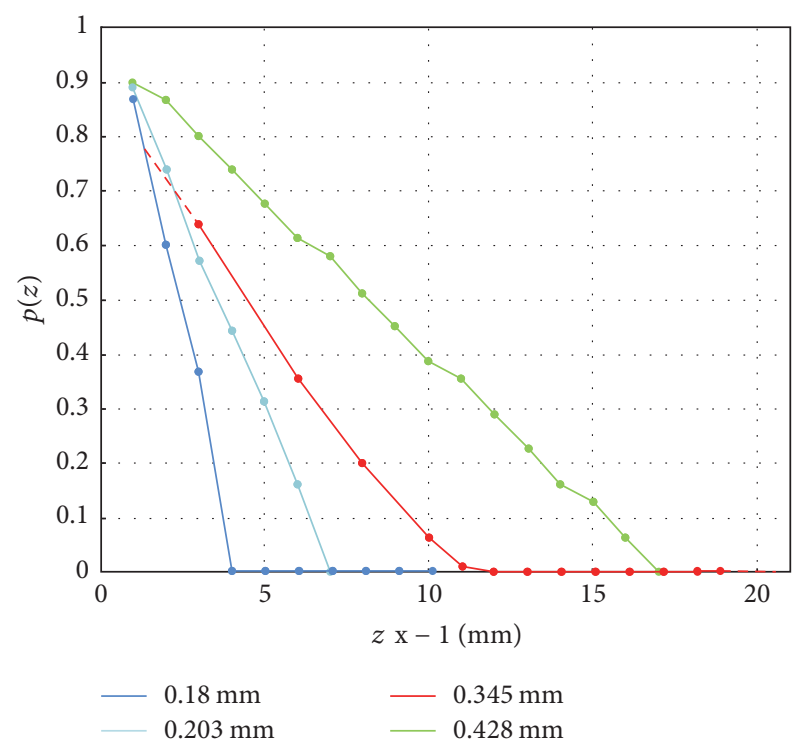

FIGURE 6: Probability $p(z)$ of the secondary electrons' passage through the penetration channel from depth $z$ for channels with differing radii.

The relationships were calculated for four values of the penetration channel's average radius $(0.18 \mathrm{~mm}, 0.203 \mathrm{~mm}$, $0.345 \mathrm{~mm}$, and $0.428 \mathrm{~mm}$ ). For each of the calculations, $\Delta l_{0}$ was taken to be $0.1 \mathrm{~mm}$ in the upper part of the penetration channel. Based on the requirement that the area of element $\Delta S$ remains constant as depth $z$ changes, the value of $\Delta l$ was calculated as $\Delta l=\Delta l_{0} \cdot r_{0} / r$, where $r_{0}$ is the radius of the penetration channel at the top.

Figure 6 presents the probability $p(z)$ of the secondary electrons' passage through the penetration channel from depth $z$. 
The plots in Figures 5 and 6 demonstrate that the magnitude of the response $\Delta I_{k}$ or the probability of electron loss decreases linearly, and at some depth $z_{\max }$ they become zero. The value of $z_{\max }$ decreases as the penetration channel's transverse dimensions decrease. Given a penetration channel with an average radius of $0.18 \mathrm{~mm}$, only electrons emitted from a depth of no more than $4 \mathrm{~mm}$ reach the collector. With wider channels, electron from a depth of $15 \mathrm{~mm}$ or more hits the collector.

Knowledge of the probability $p(z)$ of the secondary electrons' passage through the penetration channel from a depth of $z$ makes it possible to calculate the change in the secondary waveform when the beam moves as it oscillates along the walls of the channel. Suppose EBW is being performed with a small oscillation of the beam, according to some trajectory with deflections both along and across the joint. Let $O X Y$ be Cartesian coordinates in the horizontal plane. The shape of the penetration channel is described by the function $z(x, y)$, where $\tau_{x}, \tau_{y}$ is the electron beam's displacement from its equilibrium position in the penetration channel, and $j_{b}\left(x-\tau_{x}, y-\tau_{y}\right)$ is the electron beam's current density distribution.

Each beam deflection $\tau_{x}, \tau_{y}$ can be associated with the secondary waveform value $I_{k}\left(\tau_{x}, \tau_{y}\right)$. According to [23], the density of emissive current from the surface of the penetration channel, $j_{t}(x, y)$, is proportional in the first approximation to the normal of the electric field's electron density $j_{b}(x, y)_{n}=j_{b}(x, y) \cos \psi$, where $\psi$ is the beam's angle of incidence on the walls of the penetration channel. If $d S$ is an infinitely small element of the surface of the penetration channel, then the current emitted from this element is

$$
\begin{aligned}
d I_{t} & =K_{t} j_{t}(x, y) d S=\frac{K_{t} j_{t}(x, y) d x d y}{\cos \psi} \\
& =K_{t e} j_{b}\left(x-\tau_{x}, y-\tau_{y}\right) d x d y,
\end{aligned}
$$

where $K_{t}, K_{t e}$ are constants.

The share of electron current emitted from the surface of element $d S$ and reaching the collector is

$$
d I_{k}=K_{t e} j_{b}\left(x-\tau_{x}, y-\tau_{y}\right) p(z) d x d y .
$$

Finally, the waveform of the secondary electron current as a function of the electron beam's displacement $\left(\tau_{x}, \tau_{y}\right)$ is described by the integral:

$$
\begin{aligned}
I_{k}\left(\tau_{x}, \tau_{y}\right)= & \int_{a}^{b} \int_{c}^{d} K_{t e} j_{b}\left(x-\tau_{x}, y-\tau_{y}\right) \\
& \cdot p(z(x, y)) d x d y,
\end{aligned}
$$

where $a, b, c$, and $d$ are the boundaries of the region under consideration in the $O X Y$ plane.

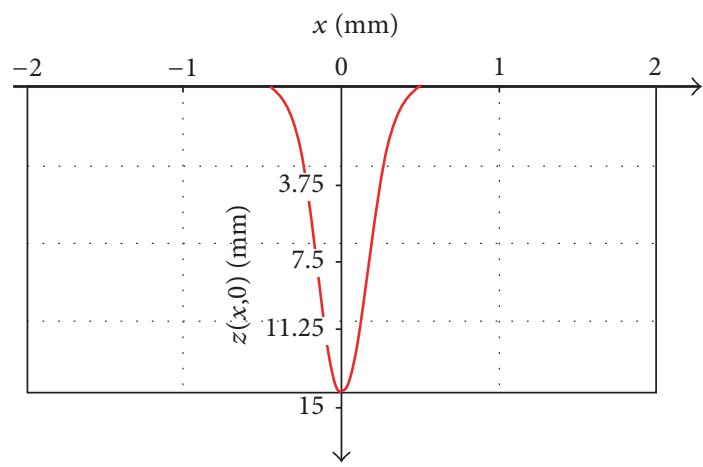

FIGURE 7: Cross-section of the penetration channel used for modeling.

We approximate the beam's current distribution and the shape of the penetration channel using standard Gaussian curves

$$
\begin{aligned}
& j_{b}(x, y)=j_{b 0} \cdot \exp \left(-\frac{x^{2}}{2 \sigma_{b x}^{2}}-\frac{y^{2}}{2 \sigma_{b x}^{2}}\right), \\
& z(x, y)=H \cdot \exp \left(-\frac{x^{2}}{2 \sigma_{x}^{2}}-\frac{y^{2}}{2 \sigma_{y}^{2}}\right),
\end{aligned}
$$

where $H$ is the penetration depth; $j_{b 0}$ is a constant that specifies the magnitude of the beam's full current; $\sigma_{x}, \sigma_{y}$ are parameters that define the width of the penetration channel; $\sigma_{b x}, \sigma_{b y}$ are parameters that define the transverse dimensions of the electron beam. We will assume $\sigma_{x}=\sigma_{y}$ and $\sigma_{b x}=\sigma_{b y}$.

We approximate the function $p(z)$ in accordance with the modeling results (Figure 6)

$$
p(z)= \begin{cases}1-\frac{z}{z_{\kappa p}}, & z \leq z_{\kappa p} \\ 0, & z>z_{\kappa p} .\end{cases}
$$

We approximate the shape of the penetration channel with a Gaussian surface. Figure 7 presents the cross-section $z(x, 0)$ of the penetration channel used for modeling.

Function $p(z(x, y))=p(x, y)$ has a zone of insensitivity (Figures 8 and 9).

For $I_{k}\left(\tau_{x}, \tau_{y}\right)$, a numeric calculation of formula (9) yields the distribution given in Figure 10. This distribution represents the change in the waveform of the secondary current in plasma when the electron beam is shifted by $\tau_{x}, \tau_{y}$ from its equilibrium position in the penetration channel. The change in the collector's current as the electron beam moves along the $x$-axis is given in Figure 11. The magnitude of the collector's current diminishes as the beam's axis approaches the axis of the penetration channel, where it reaches its minimum.

Each beam deflection corresponds to its own depth, at which the beam's center interacts with the metal on the wall of the penetration channel: $z\left(\tau_{x}, \tau_{y}\right)$. Figure 12 demonstrates the attenuation of the waveform of the collector's current as a logarithmic function of changes in this depth. The waveform's attenuation is calculated as the ratio of the current's maximum current $I_{k \max }$, which corresponds to the 


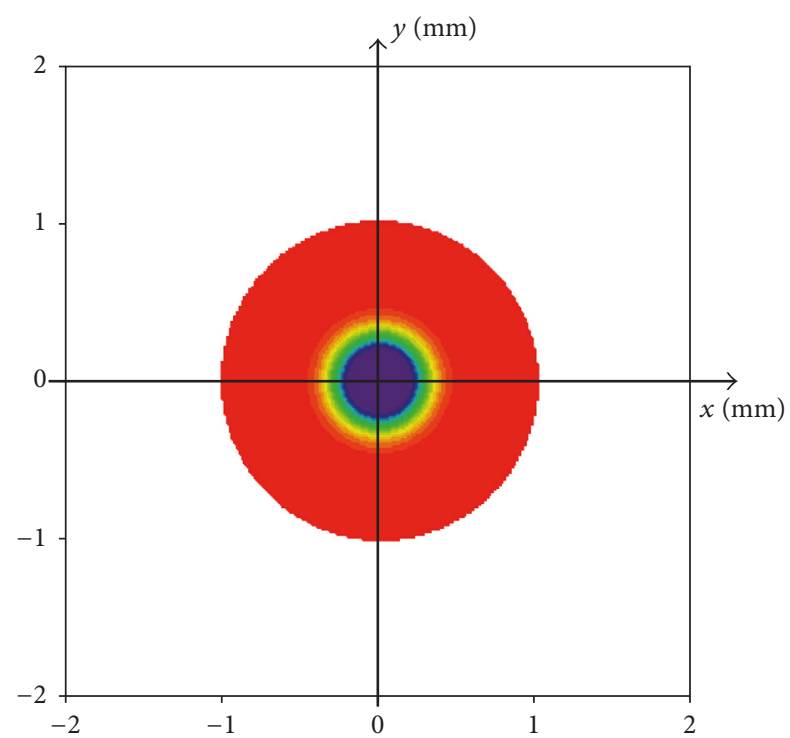

FIgURE 8: Two-dimensional distribution of the function $p(z(x, y))$.

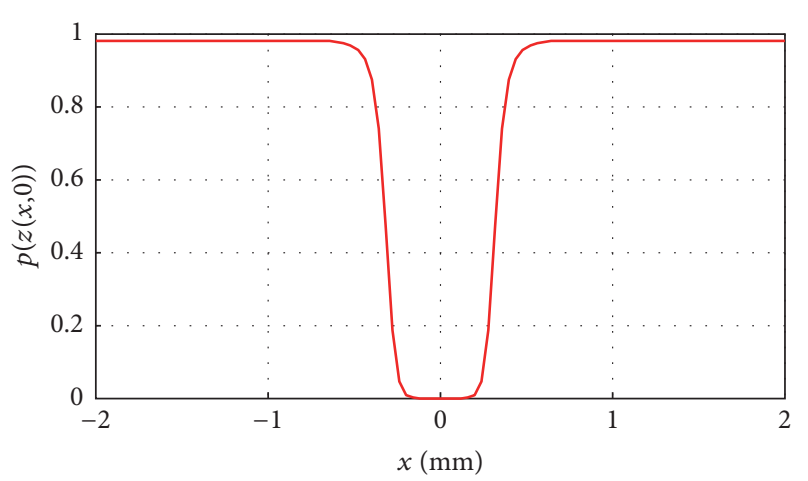

FIgURE 9: Cross-section of the function $p(z(x, y))$ when $y=0 \mathrm{~mm}$.

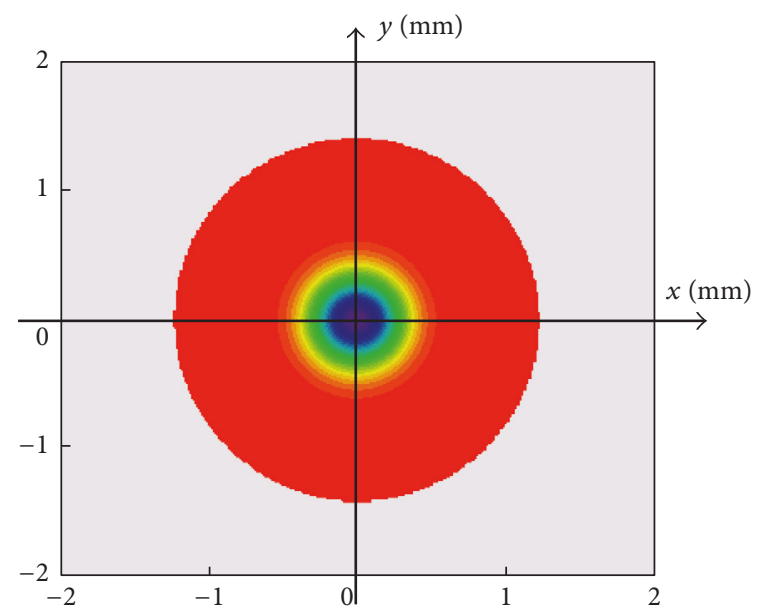

FIGURE 10: Distribution $I_{k}\left(\tau_{x}, \tau_{y}\right)$, obtained through numeric methods.

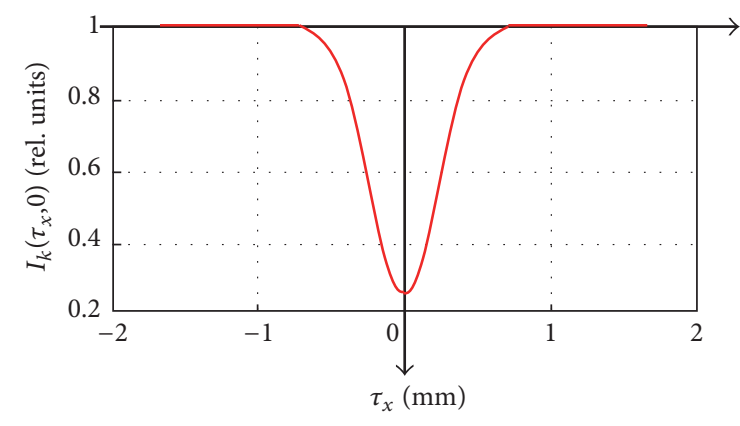

FIGURE 11: Graph of the change in the collector's current when the electron beam intersects the penetration channel as it moves along the $x$.

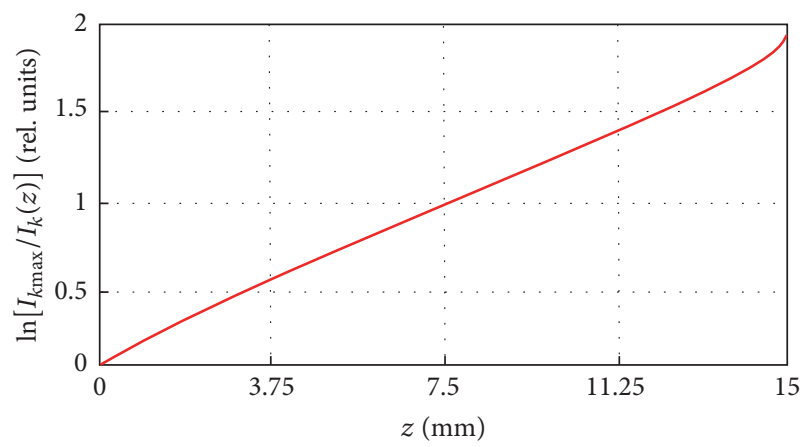

FIGURE 12: Graph of the attenuation of the collector's current as the depth of the electron beam's area of interaction with the walls of the penetration channel increases.

beam's placement on the surface of the penetration channel, to its magnitude $I_{k}(z)$ given interaction at depth $z$. Figure 12 demonstrates that over most of the interval $I_{k \max } / I_{k}(z)=$ $f(z)$ may be considered exponential.

These results confirm previous experimentally observed patterns (Figure 13).

The theoretical and experimental values are compared in Figure 14. The approximation error was $2.1 \%$. Keep in mind the fact that results calculated using formula (9) depend on the penetration channel's assumed shape. If the penetration channel has a conical shape, the error becomes $7 \%$.

\section{Conclusions}

The obtained numeric solution describes the formation of the waveform of the secondary current in plasma during changes to the penetration channel's geometric parameters. We have established that the probability of electron loss decreases linearly as depth $z$ in the channel increases, and at a certain depth $z_{\max }$ it becomes zero. We have presented a method for transitioning to a three-dimensional formulation, along with a calculation of the attenuation of the collector's current when the electron beam moves along the depth of the penetration channel. The obtained numeric solutions agree with known experimental data that indicates that the waveform has the greatest amplitude when interacting with the upper areas of the penetration channel and its amplitude decreases nearly 


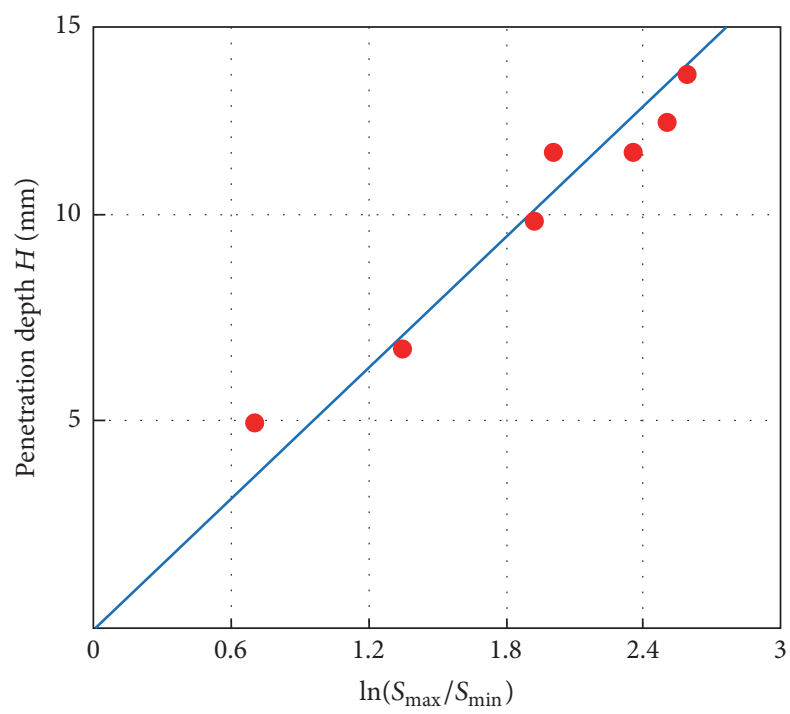

FIGURE 13: Experimental dependence between the depth of penetration and the secondary waveform parameter $\ln \left(S_{\max } / S_{\min }\right)$ given welding with oscillation along the joint and different focus settings $(P=3 \mathrm{~kW}$, oscillation frequency $f=678 \mathrm{~Hz}$, and sweep size $2 A=$ $1.3 \mathrm{~mm})[24]$.

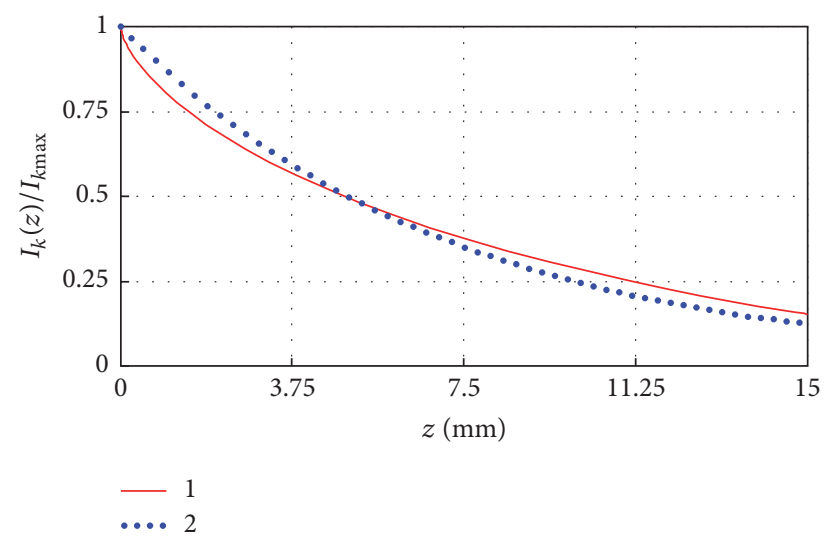

FIGURE 14: Dependence of the collector's current on the depth of the electron beam's interaction with the metal in the penetration channel. 1: theoretical curve and 2: curve based on experimental data.

exponentially along the depth of the penetration channel. These solutions may be used in researching and automating processes that process materials using concentrated energy sources.

\section{Conflicts of Interest}

The authors declare that they have no conflicts of interest.

\section{Acknowledgments}

This work was performed with the support of Grants RFFI no. 16-48-590208 r_a and State Assignment no. 9.9697.2017/BCh.

\section{References}

[1] W. Wang, S. Yamane, T. Koike et al., "Image processing method for automatic tracking of the weld line in plasma robotic welding," The International Journal of Advanced Manufacturing Technology, vol. 86, no. 5, pp. 1865-1872, 2016.

[2] I. A. Krinberg and G. M. Mladenov, "Formation and expansion of the plasma column under electron beam-metal interaction," Vacuum, vol. 77, no. 4, pp. 407-411, 2005.

[3] C. Y. Ho, M. Y. Wen, and C. Ma, "Plasma from EB evaporation of a metal target," Advances in Materials and Processing Technologies, vol. 83, pp. 190-1196, 2010.

[4] V. V. Novokreshchenov, R. V. Rodyakina, Y. V. Myakishev, and A. P. Sliva, "Determination of the degree of ionization of the vapour phase of the penetration channel in electron beam welding," Welding International, vol. 27, no. 3, pp. 238-242, 2013.

[5] V. Y. Belen'kii and V. M. Yazovskikh, "Control of electron beam welding using plasma phenomena in the molten pool region," Welding International, vol. 11, no. 7, pp. 554-556, 1997.

[6] V. Ya. Belenkii, V. M. Yazovskikh, and A. P. Zhuravlev, "Nature of the secondary current in the plasma formed in the zone affected by the electron beam during welding," Physics and Chemistry of Materials Treatment, vol.11, no. 6, pp. 128-131, 1984.

[7] G. L. Mara and R. C. McMaster, "Method for producing spikefree electron beam partial penetration welds," Welding Research Supplement, vol. 53, no. 6, pp. 246-251, 1974.

[8] R. Mayer, W. Dietrich, and D. Sundemeyer, "New high-speed beam current control and deflection system improve electron beam welding application," Welding Journal, vol. 56, no. 6, pp. 35-41, 1977.

[9] H. Schwarz, "Power density of optimally focused space-chargelimited electron beams," Journal of Applied Physics, vol. 33, no. 12, pp. 3464-3470, 1962.

[10] V. Dey, D. K. Pratihar, G. L. Datta, M. N. Jha, T. K. Saha, and A. V. Bapat, "Optimization of bead geometry in electron beam welding using a genetic algorithm," Journal of Materials Processing Technology, vol. 209, no. 3, pp. 1151-1157, 2009.

[11] U. Dilthey, A. Goumeniouk, V. Lopota, and G. Turichin, "Kinetic description of keyhole plasma in laser welding," Journal of Physics D: Applied Physics, vol. 33, no. 21, pp. 2747-2753, 2000.

[12] D. N. Trushnikov and G. M. Mladenov, "Numerical model of the plasma formation at electron beam welding," Journal of Applied Physics, vol. 117, no. 1, Article ID 013301, 2015.

[13] A. Arora, Y. V. Hote, and M. Rastogi, "Design of PID controller for unstable system," Communications in Computer and Information Science, vol. 140, pp. 19-26, 2011.

[14] D. Trushnikov, V. Belenkiy, V. Shchavlev, A. Piskunov, A. Abdullin, and G. Mladenov, "Plasma charge current for controlling and monitoring electron beam welding with beam oscillation," Sensors, vol. 12, no. 12, pp. 17433-17445, 2012.

[15] Y. P. Raizer and J. E. Allen, Gas Discharge Physics, Springer Berlin Heidelberg, Berlin, Heidelberg, 1991.

[16] A. D. Richards, B. E. Thompson, and H. H. Sawin, "Continuum modeling of argon radio frequency glow discharges," Applied Physics Letters, vol. 50, no. 9, pp. 492-494, 1987.

[17] M. A. Lieberman and A. J. Lichtenberg, Principles of Plasma Discharges and Materials Processing, John Wiley \& Sons, Inc., Hoboken, NJ, USA, 2005.

[18] R. B. Bird, W. E. Stewart, and E. N. Lightfoot, Transport Phenomena, John Wiley and Sons, 2002. 
[19] G. J. M. Hagelaar, "Modeling of microdischarges for display technology," Technische Universiteit Eindhoven, 109 pages, 2000.

[20] R. S. Brokaw, "Predicting transport properties of dilute gases," Industrial \& Engineering Chemistry Process Design and Development, vol. 8, no. 2, pp. 240-253, 1969.

[21] D. Trushnikov, E. Krotova, and E. Koleva, "Use of a secondary current sensor in plasma during electron-beam welding with focus scanning for process control," Journal of Sensors, vol. 2016, Article ID 5302681, 2016.

[22] R. Rai, T. A. Palmer, J. W. Elmer, and T. DebRoy, "Heat transfer and fluid flow during electron beam welding of 3041 stainless steel alloy," Welding Journal, vol. 88, no. 3, pp. 54-61, 2009.

[23] V. Y. Braverman, D. A. Skurikhin, S. G. Bayakin, V. F. Shabanov, and V. V. Bashenko, "A device for controlling focusing and penetration depth on the basis of inherent X-radiation in electron beam welding with modulation of the focusing level," Welding International, vol. 11, no. 7, pp. 560-563, 1997.

[24] D. N. Trushnikov, G. M. Mladenov, and V. Y. Belenkiy, "Controlling the electron beam focus regime and monitoring the keyhole in electron beam welding," Yosetsu Gakkai Ronbunshu/Quarterly Journal of the Japan Welding Society, vol. 31, no. 4, 2013. 

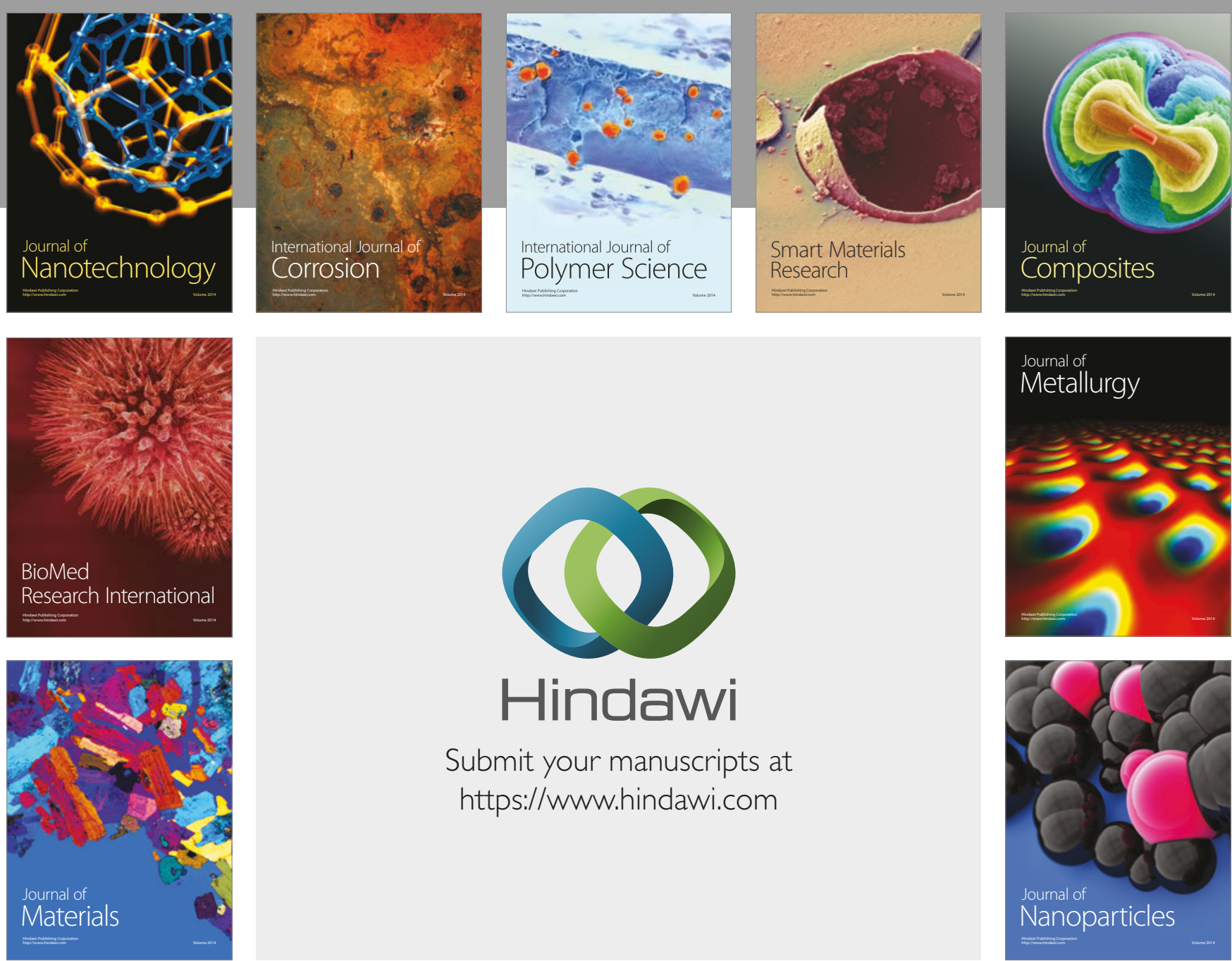

\section{Hindawi}

Submit your manuscripts at

https://www.hindawi.com
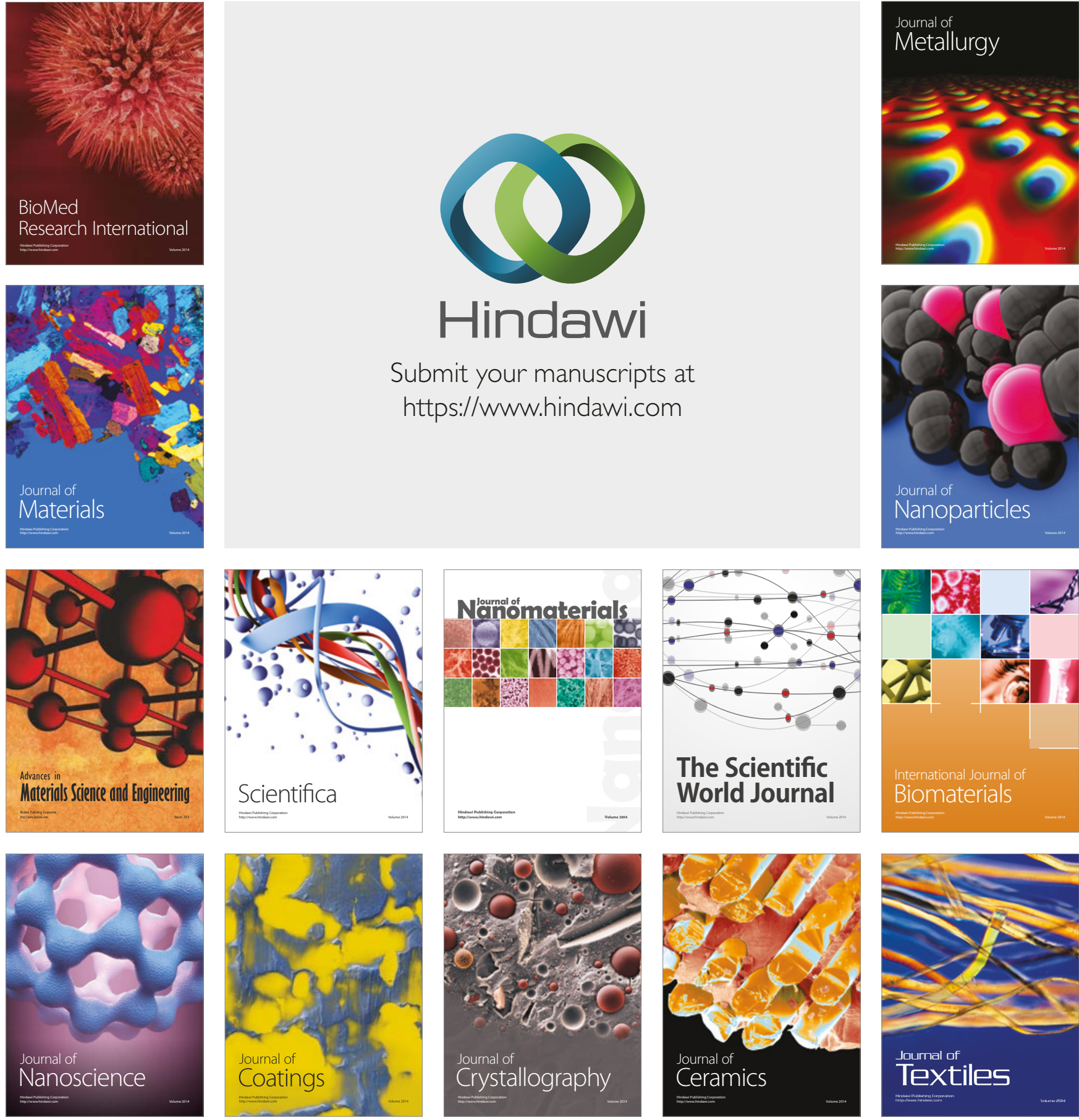

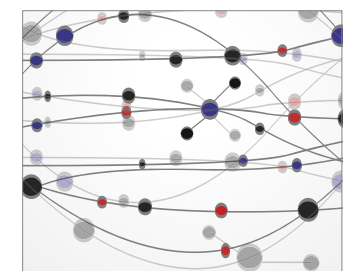

The Scientific World Journal
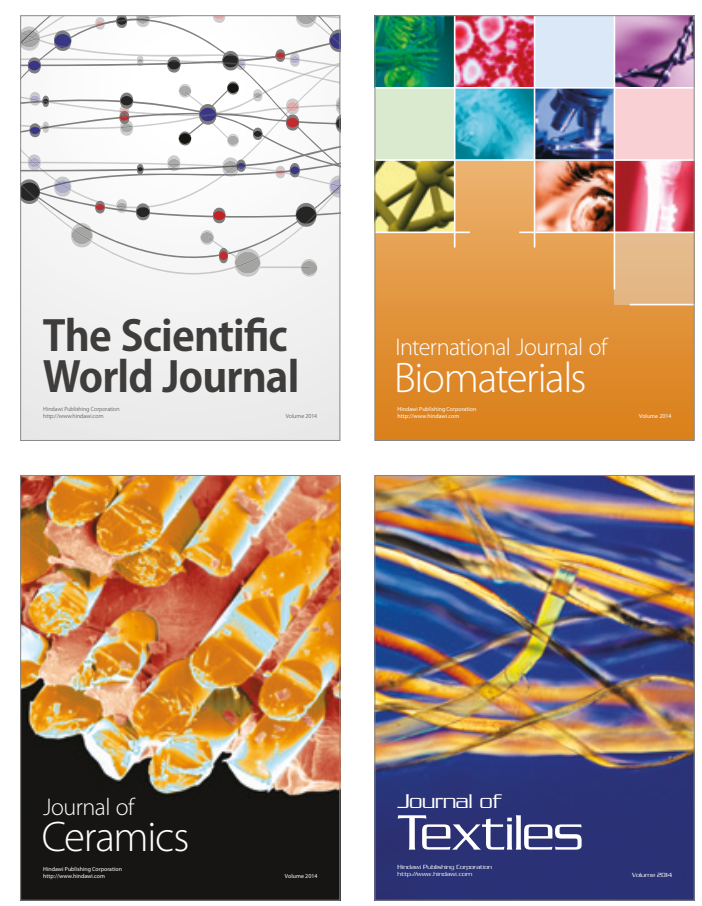